\title{
7 RELIGIOSIDADE, ESTRATÉGIAS DE COPING E SATISFAÇÃO COM A VIDA: VERIFICAÇÃO DE UM MODELO DE INFLUÊNCIA EM ESTUDANTES UNIVERSITÁRIOS
}

\author{
| Luis Fleury'; Antonio Gomes²; Julio da Rocha ${ }^{3}$; Nilton Formiga ${ }^{4}$; Marilei e Souza 5 ; Sérgio Marques ${ }^{6}$; Margarida Bernardes ${ }^{7}$ |
}

\section{RESUMO}

CONTEXTO: O ambiente universitário pode muitas vezes favorecer o desenvolvimento de problemas de saúde mental, uma vez que os universitários são submetidos a intenso nível de estresse e carga cognitiva.

OBJETIVO: Verificar a influência da religiosidade na satisfação com a vida e na adoção de estratégias para lidar com os problemas.

MÉTODOS: Estudo quantitativo com coleta de dados utilizando instrumentos psicométricos validados com 588 estudantes universitários de 18 a 63 anos. Foi utilizada a técnica de modelagem de equações estruturais para verificar um modelo de influência.

RESULTADOS: Os resultados indicam que a Religiosidade influencia positivamente e de forma significativa a Satisfação com a Vida, bem como a adoção de Estratégias para Enfrentamento de Problemas. Os resultados não são generalizáveis, entretanto constituem evidência empírica da importância da religiosidade em termos de saúde mental.

CONCLUSÃO: De forma geral, esta pesquisa pretendeu verificar um modelo teórico sistêmico, o qual hipotetizava uma interdependência entre as variáveis Religiosidade, Coping e Satisfação com a vida que se comprovou. Com isso, acredita-se que os resultados observados, teoricamente, sustentam as propostas sugeridas pela psicologia positiva.

PALAVRAS-CHAVE: Religião; Satisfação do paciente; Saúde mental

\section{RESUMEN}

"Religiosidad, estrategias de coping y satisfacción con la vida: Verificación de un modelo de influencia en estudiantes universitarios"

CONTEXTO: El ambiente universitario puede a menudo favorecer el desarrollo de problemas de salud mental, ya que los universitarios son sometidos a intenso nivel de estrés y carga cognitiva.

OBJETIVO: Verificar la influencia de la religiosidad en la satisfacción con la vida y en la adopción de estrategias para lidiar con los problemas.

MÉTODOS: Estudio cuantitativo con recolección de datos utilizando instrumentos psicométricos validados con 588 estudiantes universitarios de 18 a 63 años. Se utilizó la técnica de modelado de ecuaciones estructurales para verificar un modelo de influencia.

RESULTADOS: Los resultados indican que la Religiosidad influye positivamente y de forma significativa en la Satisfacción con la Vida, así como la adopción de Estrategias para Enfrentamiento de Problemas. Los resultados no son generalizables, sin embargo constituyen evidencia empírica de la importancia de la religiosidad en términos de salud mental.

CONCLUSIÓN: En general, esta investigación pretendió verificar un modelo teórico sistémico, el cual hipotetizaba una interdependencia entre las variables Religiosidad, Coping y Satisfacción con la vida; con ello, no sólo se comprobó el modelo pretendido, pero, se cree que los resultados observados, teóricamente, sostienen las propuestas sugeridas por la psicología positiva.

\section{DESCRIPTORES: Religión; Satisfacción del paciente; Salud mental}

\begin{abstract}
"Religiosity, coping strategies and satisfaction with life: Verification of a model of influence in university students"

BACKGROUND: The university environment can often favor the development of mental health problems, since university students are subjected to intense levels of stress and cognitive load.

AIM: To verify the influence of religiosity on satisfaction with life and on the adoption of strategies to deal with problems.

METHODS: Quantitative study with data collection using validated psychometric instruments with 588 university students aged 18 to 63 years. The structural equation modeling technique was used to verify an influence model.

RESULTS: The results indicate that Religiosity influences positively and significantly the Satisfaction with Life, as well as the adoption of Strategies to Confront Problems. The results are not generalizable, however they constitute empirical evidence of the importance of religiosity in terms of mental health.

CONCLUSION: In general, this research aimed to verify a systemic theoretical model, which hypothesized an interdependence between the variables Religiosity, Coping and Satisfaction with life; Thus, not only has the desired model been proved, but it is believed that the results observed theoretically support the proposals suggested by positive psychology.
\end{abstract}

\section{KEYWORDS: Religion; Patient satisfaction; Mental health}

Submetido em 31-03-2018

Aceite em 31-10-2018

1 Psicólogo; Mestre em Psicologia; Docente na Universidade Federal do Rio de Janeiro, Av. Pedro Calmon, 550, 23092-620 Rio de Janeiro, Brasil, luis_fleury@yahoo.com.br 2 Enfermeiro; Doutor em Enfermagem; Docente na Universidade do Estado do Rio de Janeiro, Programa de Pós-Graduação em Enfermagem, Brasil, mtosoli@gmail.com 3 Psicólogo; Doutor em Psicologia; Docente na Universidade Católica de Petrópolis, Programa de Pós-Graduação em Psicologia, Rio de Janeiro, Brasil, juliorocha@gmail.com 4 Psicólogo; Doutor em Psicologia Social; Docente na Universidade Potiguar, 23092-620 Natal, Rio Grande do Norte, Brasil, nsformiga@yahoo.com

5 Enfermeira; Doutora em Enfermagem; Docente na Universidade Severino Sombra, 23092620 Rio de Janeiro, Brasil, marileimts@hotmail.com

6 Enfermeiro; Doutor em Enfermagem; Docente na Universidade do Estado do Rio de Janeiro, Programa de Pós-Graduação em Enfermagem, sergiocmarques@uol.com.br

7 Enfermeira; Doutora em Enfermagem; Docente na Universidade do Estado do Rio de Janeiro, 23092-620 Rio de Janeiro, Brasil, margarbe@globo.com

Citação: Fleury, L. F. O., Gomes, A. M. T., Rocha, J. C. C., Formiga, N. S., Souza, M. M. T., Marques, S. C., \& Bernardes, M. M. R. (2018). Religiosidade, estratégias de coping e satisfação com a vida: Verificação de um modelo de influência em estudantes universitários. Revista Portuguesa de Enfermagem de Saúde Mental (20), 51-57. doi: 10.19131/ rpesm.0226 


\section{INTRODUÇÃO}

A questão do enfrentamento de problemas é estudada na literatura como Estratégias de Coping. Trata-se de constructo multidimensional relativo às formas como as pessoas lidam com situações estressantes (Noda, Takahashi, \& Murai, 2018). De acordo com Garrido (2018), o coping pode ser definido como o somatório dos esforços cognitivos e comportamentais que um indivíduo utiliza para lidar com uma situação de estresse que é avaliada como forte o suficiente para sobrecarregar ou exceder seus recursos pessoais. Em tais situações, o indivíduo desenvolve estratégias para lidar com as exigências que surgem.

Para Garrido (2018) o coping pode ser analisado a partir de duas óticas: foco no problema e foco na emoção. Logo, o coping com o foco no problema refere-se ao uso de estratégias que visam a atingir diretamente a fonte geradora de estresse, e causa modificações, atenuandoa ou mesmo eliminando-a, quando possível. Seriam exemplos: a negociação com pessoas envolvidas na situação, a modificação de local ou de conduta objetivando atenuar o fator estressante e a tentativa de impor limites para fazer o problema diminuir ou mesmo não se repetir.

Enquanto o foco na emoção se refere ao uso de estratégias por parte do indivíduo para lidar com a carga emocional que foi desencadeada pelo agente estressor. Seriam exemplos: a aceitação de um problema como algo natural, o esforço em manter o pensamento positivo mesmo em situação adversa, estratégias para evitar lidar com o problema e o uso de substâncias, como álcool ou mesmo drogas, como uma forma de aliviar a tensão enfrentada (Noda et al., 2018).

A questão da eficiência ou não de uma estratégia de coping deve ser avaliada com cautela. Logicamente, algumas estratégias serão mais prováveis de serem adotadas por alguns indivíduos. Outros terão preferências por estratégias diferentes, ainda que em situação semelhante.

Assim, pareceria lógico pensar que enfrentar diretamente a fonte estressora deve ser a estratégia mais eficiente, mas nem sempre isso pode ser o mais adequado, tanto pela dúvida que o indivíduo pode ter em relação à resolução ou não do problema, como em função dos custos emocionais que tal ação pode resultar. Dito de outra forma, pode ser melhor para o indivíduo se esquivar do problema do que lidar com os custos emocionais de enfrentá-lo (Delahaij \& Van Dam, 2017).
Atualmente a literatura aponta o conceito de coping como conjunto de medidas intencionais, cognitivas e comportamentais adotado pelas pessoas para adaptarem-se a diferentes circunstâncias estressantes com o propósito de minimizar sua susceptibilidade e retornar ao seu estado anterior (Melo, Carlotto, Rodriguez, \& Diehl, 2016). Ao discutir a questão do método de enfrentamento de um problema e os custos emocionais envolvidos nesse processo, traz-se à luz a satisfação do sujeito com a sua vida. Para Siqueira e Padovam (2008) a Satisfação com a Vida pode ser definida como a avaliação global feita pelo indivíduo sobre a sua vida. Sendo uma variável individual, que o indivíduo percebe como apropriada, comparando as circunstâncias da sua vida com as dispostas neste conceito. A satisfação com a vida é um julgamento cognitivo de algum ponto específico na vida da pessoa, um processo de juízo e avaliação geral da própria vida; uma avaliação sobre a vida de acordo com um critério. O julgamento da satisfação depende de uma comparação entre as circunstâncias de vida do indivíduo e um padrão por ele escolhido (Jovanovic \& Brdar, 2018). No estudo da satisfação com a vida podem ser identificados dois tipos de teorias. Aquelas que enfatizam circunstâncias objetivas e contextuais como os principais fatores na explicação da satisfação com a vida e são comumente rotuladas de teorias de baixo para cima (bottom-up theories). Por outro lado, as teorias que se baseiam em critérios individuais estáveis são comumente rotuladas de teorias de cima para baixo (top-down theories) (Samaha \& Hawi, 2016).

As teorias do tipo de cima para baixo baseiam-se na lógica de que traços da personalidade global do indivíduo explicam seu nível de satisfação com a vida. Alguns traços de personalidade em particular, como extroversão e neuroticismo, por exemplo, determinariam o grau de experiência de felicidade e satisfação com a vida dos indivíduos. A evidência da estabilidade da satisfação com a vida oferece suporte empírico a este tipo de teoria, indicando uma forte associação da satisfação com a vida com traços de personalidade e uma fraca influência do humor da pessoa, de fatores situacionais ou de eventos da vida sobre a satisfação (Samaha \& Hawi, 2016; Whisman \& Judd, 2016).

Ressalta-se que essa investigação ancora-se nos pressupostos teóricos da Psicologia Positiva ao entender que a satisfação com a vida constitui importante indicador de saúde e qualidade de vida apontado pela literatura especializada (Seligman \& Csikszentmihalyi, 2000; Antoine, Dauvier, Andreotti, \& Congard, 2018). 
Por fim considerou-se nesta pesquisa as variáveis de religiosidade e espiritualidade. $\mathrm{O}$ estudo baseou-se nos conceitos de religiosidade e espiritualidade dos principais autores mundiais que trabalham no contexto da saúde, especialmente Koenig, Pargament e Nielsen (1998) e Moreira-Almeida (2007). Para estes autores, religião e religiosidade envolvem a adoção de um conjunto de crenças, práticas e rituais, na maioria das vezes relacionadas a um ser superior e transcendente. Tratase de uma dimensão sociológica, de vivência e convivência comunitária. A espiritualidade, por sua vez, pode ser definida como uma conexão com o sagrado e o transcendente, bem como pela busca de sentido, não necessariamente mediada pela religião, mas passando por ela para uma grande maioria de pessoas.

Atualmente, as relações entre religiosidade e saúde física e/ou mental - vêm sendo crescentemente investigadas.

A maior parte dos estudos indica que a religiosidade é habitualmente um fator de proteção que deve ser observado na prática clínica contra o desenvolvimento de transtornos mentais (por exemplo, depressão, ansiedade, abuso de substâncias, etc.), além de estar associada a melhor qualidade de vida (Taunay et al., 2012; Bravo, Pearson, \& Stevens, 2016).

\section{MÉTODOS}

Participaram do estudo 588 universitários, de 18 a 63 anos ( $\mathrm{M}=25,01$ ano, $\alpha=12,03$ anos), com a prevalência de mulheres (81\%), a maioria (58\%) tem uma renda econômica entre 1 e 3 salários mínimos.

Esta amostra é não probabilística e de conveniência, pois foi considerada a pessoa que, consultada, dispusera-se a colaborar respondendo ao questionário que era apresentado. Para estabelecer a significância da amostra, esta foi calculada no pacote estatístico G Power 3.1 que é um software destinado a calcular o poder estatístico - o teste de hipótese, tendo como base, não apenas o 'n' necessário para a pesquisa, mas, também, o tipo de cálculo a ser realizado (Faul, Erdfelder, Lang, \& Buchner, 2007).

Para a coleta de dados deste estudo, considerando uma probabilidade de $95 \%$ ( $p<0,05)$, magnitude do efeito amostral $(r \geq 0,50)$ e um padrão de poder hipotético $(\pi$ $\geq 0,80$ ); a amostra especificada revelou-se suficiente $\mathrm{e}$ significativa (tendo como indicadores: $t \geq 1,98 ; \pi \geq 1,00$; $\mathrm{p}<0,05)$.

Os participantes responderam aos seguintes instrumentos:
- Caracterização Sócio-demográfica. Foram elaboradas perguntas que contribuíram para identificar os participantes deste estudo (por exemplo, sexo, idade, estado civil, renda econômica, escolaridade, bem como, realizar um controle estatístico de algum atributo que possa interferir diretamente nos seus resultados.

- Inventário Multifatorial de Coping (IMCA), de autoria de Antoniazzi (2000). Tal instrumento é constituído por 43 itens, divididos em quatro diferentes estratégias de enfrentamento de situações estressantes: ação direta, ação social, negação e autocontrole. O fator "ação direta" é constituído por 14 itens, definida como estratégias que apresentam ações e comportamentos que buscam modificar as circunstâncias e os eventos estressantes, sendo, portanto, considerada uma estratégia eficaz e benéfica ao indivíduo. A "ação social” é o segundo fator do instrumento, a qual implica criar estratégias ligadas à busca por auxílio externo para a resolução dos conflitos, buscar familiares e amigos próximos para obter ajuda, como conselhos e conforto. $\mathrm{O}$ fator é formado por 8 itens. O terceiro fator do IMCA é denominado “negação". Ele é constituído por 11 itens que juntos se referem à tentativa de o indivíduo de não enxergar o problema, e é considerado como uma estratégia ineficiente e nociva tanto ao indivíduo quanto ao combate ao problema em si; afinal, inicialmente, faz com que o indivíduo mude o olhar, o foco da situação estressante, sem, contudo, deixar de percebê-la. O quarto e último fator da escala é denominado "autocontrole", o qual é aferido por 10 itens relacionados a estratégias de enfrentamento de problemas estressantes, quando o indivíduo busca manter a calma e se controlar, de modo a se estressar menos. O índice de confiabilidade (Alfa de Cronbach) do instrumento foi igual a 0,845 .

- A satisfação com a vida (SV) foi mensurada com a utilização da Escala de Satisfação com a Vida validada para amostras brasileiras por Gouveia, Barbosa, Andrade e Carneiro (2005). A escala é composta por 5 itens que dizem respeito a percepção geral do quanto o sujeito está satisfeito com a sua vida. Neste estudo, observou-se uma alfa de 0,780.

- Escala de Religiosidade da Duke - DUREL (ERD), composta por 5 itens, validada por Taunay et al. (2012). A escala é composta por 5 itens que dizem respeito a frequência que o sujeito vai ao templo religioso, frequência que o sujeito dedica a atividades religiosas individuais e sobre as suas experiências religiosas. No presente estudo, a escala em questão, apresentou um alfa de 0,84 e um ICC (Coeficiente de Correlação Intraclasse) de 0,86 (95\% IC variando de 0,85-0,88; F = $7,37, \mathrm{p}<0,005)$, condição essa, que não interfere na consistência da medida da ERD em questão. 


\section{Procedimentos Éticos}

Todos os procedimentos adotados nesta pesquisa seguiram as orientações éticas previstas na Resolução 196/96 do CNS e da Resolução 016/2012 do Conselho Federal de e Psicologia. E estão de acordo com a Resolução 510/2016 do Conselho Nacional de Saúde para pesquisas realizadas com amostras de seres humanos. Os responsáveis pela coleta dos dados visitaram diferentes universidades. Foi dito aos participantes que não havia resposta certa ou errada e que mesmo necessitando uma resposta individual, estes não deveriam se ver obrigados em respondê-los podendo desistir a qual momento seja quanto tivesse o instrumento em suas mãos ou ao iniciar sua leitura, ou outra eventual condição. Todos assinaram o Termo de Consentimento Livre e Esclarecido. Em qualquer um desses eventos, não haveria problema de sua desistência. A todos era assegurado o anonimato das respostas e que estas seriam tratadas em seu conjunto estatisticamente.

\section{Análise de Dados}

Utilizou-se a versão 21.0 do pacote estatístico SPSS para Windows, e computadas estatísticas descritivas. E o software AMOS GRAFICS, versão 21.0, para o Modelo de Equações Estruturais (MEE), sendo considerados os seguintes índices para ajuste do modelo de acordo com a literatura especializada (Hair, Tatham, Anderson \& Black, 2009):

- O $\chi^{2}$ (qui-quadrado) testa a probabilidade de o modelo teórico se ajustar aos dados; quanto maior este valor pior o ajustamento. É comum considerar sua razão em relação aos graus de liberdade $\left(\chi^{2} / g .1.\right)$. Neste caso, valores até 5 indicam um ajustamento adequado;

- Root Mean Square Residual (RMR), que indica o ajustamento do modelo teórico aos dados, na medida em que a diferença entre os dois se aproxima de zero. Para o modelo ser considerado bem ajustado, o valor deve ser menor que 0,05 ;

- O Goodness-of-Fit Index (GFI) e o Adjusted Goodness-of-Fit Index (AGFI) são análogos ao $\mathrm{R}^{2}$ em regressão múltipla. Portanto, indicam a proporção de variância-covariância nos dados explicada pelo modelo. Estes variam de 0 a 1, com valores na casa dos 0,80 e 0,90 , ou superior, indicando um ajustamento satisfatório.

- A Root-Mean-Square Error of Approximation (RMSEA), com seu intervalo de confiança de 90\% (IC90\%), é considerado um indicador de desajuste, isto é, valores altos indicam um modelo não ajustado. Assumese como ideal que o RMSEA se situe entre 0,05 e 0,08, aceitando-se valores de até 0,10 ;
- O Comparative Fit Index (CFI), compara de forma geral o modelo estimado e o modelo nulo, considerando valores mais próximos de um como indicadores de ajustamento satisfatório;

- O Normed Fit Index (NFI), varia de zero a um e pode ser considerado aceitável para valores superiores a 0,90 . Caracteriza-se por ser uma medida de comparação entre o modelo proposto e o modelo nulo, representando um ajuste incremental.

\section{RESULTADOS}

Para atender ao objetivo principal do presente estudo que é investigar a influência da Religiosidade sobre a Satisfação com a Vida e Coping, considerou-se um modelo recursivo de equações estruturais. Observou-se após a realização das devidas modificações de ajustes, que o modelo proposto se mostrou adequado, uma vez que os constructos avaliados apresentaram uma associação interdependente (Tabela 1). Neste modelo é possível destacar os seguintes índices estatísticos: $\left(\mathrm{x}^{2} / \mathrm{gl}=\right.$ 1,97; RMR = 0,05; GFI = 0,98; $\mathrm{AGFI}=0,95$; $\mathrm{CFI}=0,98$; $\mathrm{NFI}=0,95$ e RMSEA $(90 \% \mathrm{IC})=0,04(0,03-0,05)$.

Tabela 1 - Associações Lambdas $(\gamma)$ entre os Constructos do Modelo Teórico

\begin{tabular}{|l|l|l|l|}
\hline & Religiosidade & $\begin{array}{l}\text { Satisfação } \\
\text { com a vida }\end{array}$ & Coping \\
\hline Religiosidade & \multicolumn{1}{|c|}{--} & & \\
\hline Satisfação com a vida & 0,31 & --- & \\
\hline Coping & 0,38 & 0,36 & --- \\
\hline
\end{tabular}

Considerando esses resultados, o modelo apresentou indicadores aceitáveis de acordo com o que exigido na literatura vigente (Hair et al., 2009). No que se refere ao modelo gerado, vale destacar que todas as saturações (Lambdas, $\gamma$ ) estão dentro do intervalo esperado $\mid 0$ - 1|, e denotam não haver problemas da estimação proposta, pois todas foram estatisticamente diferentes de zero $(\mathrm{t}$ $>1.96, \mathrm{p}<0.05)$. Ao analisar os resultados supracitados, com base nas estimativas de predição entre as associações das variáveis e a partir da análise de regressão referente ao modelo teórico elaborado, observou-se, que todas as variáveis foram significativas a um $\mathrm{p}<$ 0,001 (Tabela 2). 
Tabela 2 - Indicadores das Estimativas

Preditivas do Modelo Teórico Hipotetizado

\begin{tabular}{|l|l|l|l|l|l|l|l|}
\hline $\begin{array}{l}\text { Con- } \\
\text { structo }\end{array}$ & Variáveis & $\begin{array}{l}\text { Esti- } \\
\text { mativa }\end{array}$ & d.p. & $\begin{array}{l}\text { Razão } \\
\text { Crité- } \\
\text { rio }\end{array}$ & P & VIF \\
\hline cop & $<---$ & rel & 0,56 & 0,02 & 2,76 & 0,001 & 1,00 \\
\hline stv & $<---$ & rel & 0,71 & 0,03 & 6,13 & 0,001 & 1,07 \\
\hline stv & $<---$ & cop & 0,66 & 0,09 & 5,80 & 0,001 & 1,01 \\
\hline sats1 & $<---$ & stv & 1,00 & --- & --- & --- & 1,98 \\
\hline sats2 & $<---$ & stv & 0,86 & 0,06 & 15,16 & 0,001 & 1,54 \\
\hline sats3 & $<---$ & stv & 0,94 & 0,05 & 17,45 & 0,001 & 1,81 \\
\hline sats4 & $<---$ & stv & 0,69 & 0,05 & 13,38 & 0,001 & 1,37 \\
\hline sats5 & $<---$ & stv & 0,77 & 0,06 & 11,06 & 0,001 & 1,25 \\
\hline reli5 & $<---$ & rel & 1,00 & --- & --- & --- & 2,03 \\
\hline reli4 & $<---$ & rel & 0,82 & 0,04 & 19,37 & 0,001 & 1,87 \\
\hline reli3 & $<---$ & rel & 0,71 & 0,04 & 17,02 & 0,001 & 1,86 \\
\hline reli2 & $<---$ & rel & 0,99 & 0,06 & 15,26 & 0,001 & 2,10 \\
\hline reli1 & $<---$ & rel & 0,99 & 0,06 & 16,32 & 0,001 & 2,53 \\
\hline $\begin{array}{l}\text { Ação- } \\
\text { Direta }\end{array}$ & $<---$ & cop & 1,000 & --- & --- & --- & 1,37 \\
\hline $\begin{array}{l}\text { Ação- } \\
\text { Social }\end{array}$ & $<---$ & cop & 0,88 & 0,05 & 17,66 & 0,001 & 1,08 \\
\hline $\begin{array}{l}\text { Auto- } \\
\text { Con- } \\
\text { trole }\end{array}$ & $<---$ & cop & 0,74 & 0,12 & 15,55 & 0,001 & 1,35 \\
\hline $\begin{array}{l}\text { Nega- } \\
\text { ção }\end{array}$ & $<---$ & cop & 0,67 & 0,04 & 12,65 & 0,001 & 1,06 \\
\hline Notas: & Reigis & & & & & & \\
\hline
\end{tabular}

Notas: rel $=$ Religiosidade; stv $=$ Satisfação com vida cop $=$ Coping; sats 1 ...sats $5=$ itens da escala de satisfação com vida; reli1 ...reli5 = itens da escala de religiosidade.

É preciso destacar que, nestes resultados, não encontrou-se multicolineariedade, condição essa, observada no cálculo de Inflação da Variância do Fator (IVF) que baseia-se no fator de tolerância entre as variáveis (este índice, deve apresentar escores que não excedam um valor acima de 5), pois implica na baixa qualidade do modelo empírico estimado (Hair et al., 2009), este indicador variou na presente análise de 1,00 a 2,53 ( $\mathrm{p}<$ $0,01)$.

É possível afirmar que o modelo teórico que hipotetiza a influência da religiosidade na satisfação com a vida e na adoção de estratégias de coping foi comprovado, uma vez que, além de apresentar indicadores psicométricos adequados quanto à modelagem estrutural aceita na literatura estatística, revelou uma associação positiva entre os constructos (Hair et al., 2009). Com isso, de forma geral tais resultados permitem afirmar a influência da variável religiosidade sobre coping e satisfação com a vida.

\section{DISCUSSÃO}

De forma geral, esta pesquisa pretendeu verificar um modelo teórico sistêmico, o qual hipotetizava-se uma interdependência entre as variáveis Religiosidade, Coping e Satisfação com a Vida. Dessa maneira, não apenas se comprovou o modelo pretendido, mas, acredita-se que os resultados observados estão dentro do pressuposto teórico da Psicologia Positiva (Antoine et. al., 2018).

Assim, pode-se refletir em relação ao funcionamento sistêmico do sujeito onde habilidades, potenciais humanos, motivações e recursos adaptativos devem ser fomentados no tratamento clínico para que esse indivíduo tenha melhores condições de saúde mental e consequentemente sentir-se mais capaz de lidar com as demandas do ensino universitário (Antoine et. al., 2018). Pôde-se verificar através das análises realizadas que a religiosidade é uma importante variável a ser desenvolvida e que pode influenciar o desenvolvimento da satisfação com a vida.

Nestes termos, o estudo corrobora o que a literatura relata sobre o funcionamento da religiosidade na saúde do sujeito (Moreira-Almeida, 2007; Taunay et al., 2012). Bem como, corrobora com a importância da avaliação do coping para investigação de fenômenos relativos a saúde mental, sobretudo no contexto universitário que é um ambiente propício ao desenvolvimento de estresse pela elevada carga cognitiva a que os estudantes são submetidos (Garrido, 2018; Delahaij \& Van Dam, 2017).

Por fim, ressalta-se que a Psicologia Positiva objetiva o desenvolvimento, nas práticas clínicas de cuidado com a saúde mental, de potencialidades do sujeito em contraposição aos desafios que o indivíduo precisa lidar na sua rotina.

Sendo assim, o desenvolvimento de um ambiente que proporcione satisfação com a vida e o incentivo a vivência das práticas religiosas individuais devem ser fomentadas pelo contexto universitário e também pelos profissionais de saúde em suas práticas clínicas a fim de lidar com a estressante rotina universitária que pode levar aos alunos ao adoecimento e evasão (Jovanovic \& Brdar, 2018; Samaha \& Hawi, 2016; Whisman \& Judd, 2016; Garrido, 2018; Machado, Gurgel \& Reppold, 2017). 


\section{CONCLUSÕES}

A principal conclusão deste estudo é que a religiosidade é uma importante aliada no desenvolvimento da satisfação com a vida e que esse fenômeno exerce influência na adoção da estratégia a ser utilizada para o enfrentamento de um problema.

Uma limitação deste estudo é que não se pode generalizar os resultados, mas embora os resultados estejam relacionados a esse contexto amostral, indicam importantes evidências empíricas do funcionamento da religiosidade para formação e desenvolvimento saudável do sujeito.

Sugere-se para estudos futuros, que se realize uma comparação entre universitários que relatem práticas religiosas com aqueles que não relatam a mesma vivência religiosa.

Assim, será possível estudar o desempenho em variáveis relacionadas a saúde de sujeitos religiosos e não religiosos.

\section{IMPLICAÇÕES PARA A PRÁTICA CLÍNICA}

O presente estudo deixa claro que o profissional de saúde deve utilizar em sua prática clínica a religiosidade e espiritualidade presente no sujeito como aliadas no tratamento de enfermidades mentais o que está em conformidade com a literatura consultada (Bravo et al., 2016).

Logo, não se pode negligenciar o papel estratégico que a religiosidade e a espiritualidade pode ter em uma intervenção de saúde no contexto do adoecimento relacionado a vida universitária. Desse modo, os gestores responsáveis pela saúde no âmbito universitário podem fomentar a criação de espaços que acolham grupos para o desenvolvimento de práticas religiosas na universidade.

Tais grupos podem atuar como aliados no tratamento e na adesão ao tratamento proposto pelo centro de saúde, bem como podem acompanhar o desenvolvimento das práticas terapêuticas propostas pelos profissionais de saúde.

Essa proposta se sustenta com base nas evidências apontadas neste artigo de que uma vivência da religiosidade e da espiritualidade estão associadas a maior satisfação com a vida e a estratégias de enfrentamento de problemas mais saudáveis.

\section{REFERÊNCIAS BIBLIOGRÁFICAS}

Antoine, P., Dauvier, B., Andreotti, E., \& Congard, A. (2018). Individual differences in the effects of a positive psychology intervention: Applied psychology. Personality and Individual Differences, 122, 140-147. Doi: 10.1016/j.paid.2017.10.024

Antoniazzi, A. S. (2000). Desenvolvimento de instrumentos para a avaliação de coping em adolescentes brasileiros. (Tese de Doutoramento). Universidade Federal do Rio Grande do Sul, Brasil.

Bravo, A. J., Pearson, M. R., \& Stevens, L. E. (2016). Making religiosity person-centered: A latent profile analysis of religiosity and psychological health outcomes. Personality and Individual Differences, 88, 160169. Doi: 10.1016/j.paid.2015.08.049

Delahaij, R. \& Van Dam, K. (2017). Coping with acute stress in the military: The influence of coping style, coping self-efficacy and appraisal emotions. Personality and Individual Differences, 119, 13-18. Doi: 10.1016/j. paid.2017.06.021

Faul, F., Erdfelder, E., Lang, A. G., \& Buchner, A. (2007). $\mathrm{G}^{\star}$ Power 3: A flexible statistical power analysis program for the social, behavioral, and biomedical sciences. Behavior research methods, 39 (2), 175-191.Disponível em: https://www.ncbi.nlm.nih.gov/pubmed/17695343

Garrido, S. (2018). The influence of personality and coping style on the affective outcomes of nostalgia: Is nostalgia a healthy coping mechanism or rumination? Personality and Individual Differences, 120, 259-264. Doi: $10.1016 /$ j.paid.2016.07.021

Gouveia, V. V., Barbosa, G. A., Andrade, E. O., e Carneiro, M. B. (2005). Medindo satisfação com a vida dos médicos no Brasil. Jornal Brasileiro de Psiquiatria, 54 (4), 298-305. Disponível em: http://pesquisa.bvsalud. org/portal/resource/pt/lil-438324

Hair, J. F., Tatham, R. L., Anderson, R. E., \& Black, W. (2009). Análise Multivariada de dados. (6 $6^{\mathrm{a}}$ ed.). Porto Alegre: Bookman. 
Jovanovic, V., \& Brdar, I. (2018). The cross-national measurement invariance of the Satisfaction with Life Scale in a sample of undergraduate students. Personality and Individual Differences, 128, 7-9. Doi: 10.1016/j. paid.2018.02.010

Koenig, H. G., Pargament, K. I., \& Nielsen, J. (1998). Religious coping and health status in medically ill hospitalized older adults. The Journal of Nervous and Mental Disease, 186, 513-521. Disponível em: https://www. ncbi.nlm.nih.gov/pubmed/9741556

Machado, F. A., Gurgel, L. G., \& Reppold, C. T. (2017). Intervenções em Psicologia Positiva na reabilitação de adultos e idosos: revisão da literatura. Estudos de Psicologia (Campinas), 34 (1), 119-130. Doi: 10.1590/198202752017000100012

Melo, L. P., Carlotto, M. S., Rodriguez, S. Y. S., e Diehl, L. (2016). Estratégias de enfrentamento (coping) em trabalhadores: revisão sistemática da literatura nacional. Arquivos Brasileiros de Psicologia, 68 (3), 125-144. Disponível em: http://pepsic.bvsalud.org/scielo.php?script=sci_artt ext\&pid=S1809-52672016000300010

Moreira-Almeida, A. (2007). Espiritualidade e saúde: passado e futuro de uma relação controversa e desafiadora. Revista de Psiquiatria Clínica, 34 (1). Doi: 10.1590/ S0101-60832007000700001

Noda, T., Takahashi, Y., \& Murai, T. (2018). Coping mediates the association between empathy and psychological distress among Japanese workers. Personality and Individual Differences, 124, 178-183. Doi: 10.1016/j. paid.2017.12.009
Samaha, M., \& Hawi, N. S. (2016). Relationships among smartphone addiction, stress, academic performance, and satisfaction with life. Computers in Human Behavior, 57, 321-325. Doi: 10.1016/j.chb.2015.12.045

Seligman, M. E. P. \& Csikszentmihalyi, M. (2000). Positive Psychology: an introduction. American Psychologist, 55 (1), 5-14. Doi: 10.1037/0003-066X.55.1.5

Siqueira, M. M. M., e Padovam, V. A. R. (2008). Bases Teóricas de Bem-Estar Subjetivo, Bem-Estar Psicológico e Bem-Estar no Trabalho. Psicologia: Teoria e Pesquisa, 24 (2), 201-209. Disponível em: http://www.scielo.br/scielo.php?pid=S0102-37722008000200010\&script $=$ sci_ abstract\&tlng=pt

Taunay, T. C. D., Aquino, F. A., Gondim, D. S. M., Moreira-ALmeida, A., Gurgel, L. A., Andrade, L., e Carvalho, A. F. (2012). Validação da versão brasileira da escala de religiosidade de Duke (DURELL). Revista de Psiquiatria Clínica, 39 (4), 130-135. Doi: 10.1590/ S0101-60832012000400003

Whisman, M. A. \& Judd, C. M. (2016). A cross-national analysis of measurement invariance of the Satisfaction With Life Scale. Psychological Assessment, 28 (2), 239244. Doi: $10.1037 /$ pas0000181

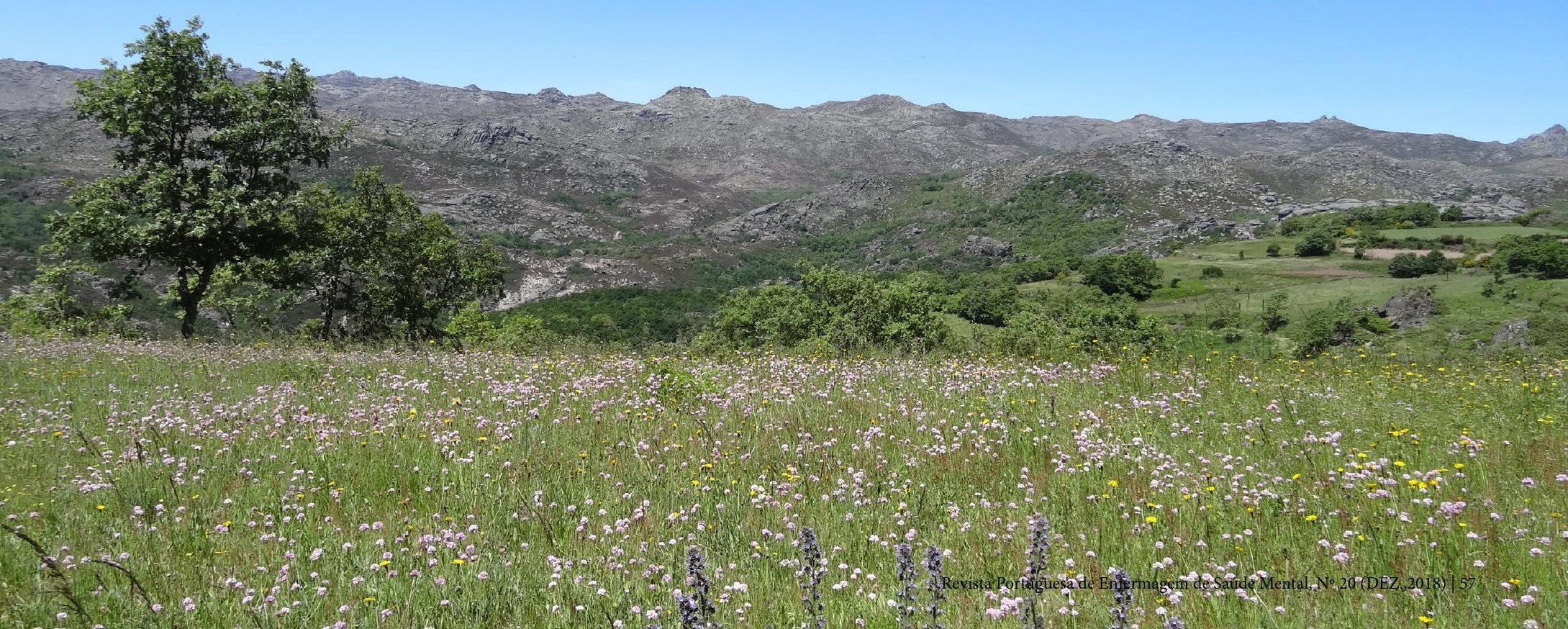

